AperTO - Archivio Istituzionale Open Access dell'Università di Torino

\title{
Evaluative Morphology and Sociolinguistic Variation
}

\section{This is a pre print version of the following article:}

Original Citation:

\section{Availability:}

This version is available http://hdl.handle.net/2318/1532443

since 2015-12-10T12:53:28Z

Publisher:

Edinburgh University Press

Terms of use:

Open Access

Anyone can freely access the full text of works made available as "Open Access". Works made available under a Creative Commons license can be used according to the terms and conditions of said license. Use of all other works requires consent of the right holder (author or publisher) if not exempted from copyright protection by the applicable law. 


\title{
9 Evaluative Morphology and Sociolinguistic Variation
}

\author{
Livio Gaeta
}

\section{Introduction}

Word-formation rules have been shown to be generally sensitive to registers and text types (see Baayen 1994 on English). For instance, spoken varieties of English are said to display poorer word formation than the written language (Plag, Dalton-Puffer and Baayen 1999). This is particularly true of those word-formation rules whose meaning has a less concrete content, such as processes forming denominal adjectives, deadjectival or deverbal nouns, and so on. In other words, word-formation rules displaying a more 'grammatical' and less 'referential' meaning are expected to be less used in spoken, informal varieties. At any rate, few studies have been devoted to investigating systematically the exact extent of this assumption. While Roelcke $(1999,81)$ observes a significant increase of nominalisations in the written variety of German for special purposes in contrast with the standard - including the spoken - varieties, Iacobini and Adinolfi (2008) find no significant differences comparing written and spoken varieties of Italian. Unfortunately, since they did not investigate any evaluative morphology (EM), their results are useless for our purposes. Plag et al. (1999) fare slightly better because - in spite of the very poor EM of English - they included in their suffix sample -ish, which proved to be used significantly more extensively in everyday conversations than in other more controlled varieties and in the written language. Moreover, Grandi (2003a) finds that the Italian augmentative suffix -one is more frequently used in written texts mimicking spoken varieties like comedies or in those parts of narrative texts reproducing spoken situations (typically dialogues), especially when characters of humble origin are involved. This provides support for pointing out an inverse relation: while the usage frequency of more grammatical word-formation processes, measured in terms of both types and tokens, generally increases as long as the register and/or the text type show(s) an increasing degree of formality, the opposite is true for EM. In what follows we will try to survey the different factors influencing the usage of EM, ranging from text types to social contexts and socio-pragmatic environments.

\section{The role of sociolinguistic factors in evaluative morphology}

As is well known, sociolinguistic variation relates to several domains and calls into play different factors influencing the language in its concrete use and ultimately shaping the speech act. Here, we will distinguish two different perspectives. On the one hand, we will discuss the role that EM plays in contributing to the general sociolinguistic perspective of identifying the social characters of the speakers involved in a given speech situation. In 
this light, the traditional (especially diastratic and diaphasic) dimensions of variation will be considered. Under the diastratic heading, all truly sociological variables are considered, such as social group or class, ethnic background, sex, age and so on. Only marginal reference will be made to the diatopic dimension because that is more related to a truly dialectal variation. As for the so-called diamesic perspective, relating to the communication channel (see Berruto 1995, 123 for discussion), it rather characterises specific linguistic varieties and only indirectly provides information on the speakers' social attitudes and characters. For this reason, it will be mainly considered in relation to the diaphasic dimension as long as the latter is concerned with the speakers' field of activity, their working environment and so on, besides the speakers themselves in their own personal sphere of social interaction. In this regard, the enormous increase of written modalities of social interaction recorded in the last decades thanks to the diffusion of the Internet, social networks and so on promises to increase significantly the role played by the diamesic dimension in contributing to the identification of the social characters of the speakers involved in such communicative situations. In other words, the use of certain communicative modalities and their typical linguistic features are likely to provide substantive information on the social character of the speakers using them, and this on a mass scale by virtue of the large user numbers.

On the other hand, we will try to see briefly how much EM (as an instantiation of the general domain of word formation) is utilised more or less consciously by the speakers to carry out a number of operations on the speech act which reveal their social character or attitude. This opens the door for the consideration of the morphopragmatic dimension as understood by Dressler and Merlini Barbaresi (1994).

\subsection{Sociolinguistic factors in the use of evaluative morphology}

One general question concerns the real possibility for EM to be influenced by sociolinguistic factors. In fact, it is often claimed that only some aspects of language are likely to be exposed to the influence of the (socially or pragmatically determined) extra-linguistic context, for instance those relating to deixis (Fasold 1992, 352). In this case, contextual extra-linguistic - for instance, spatial, perceptual and so on - factors arguably guide the way language concretely encodes reality, and conversely how the latter has an impact on the language structure. On the other hand, other aspects of language more related to the grammar intended as a description of our language competence are normally independent of the context, in the specific sense that language-internal structural relations are not subject to its influence (Romaine 1984, 34). Among the latter one usually includes besides phonology and syntax - morphology and word formation, although the latter often goes unmentioned. Clearly, this does not exclude the possibility that (evaluative) morphology may carry context-related or more general social meaning, but it is generally held that morphological markers cannot be assigned a direct extra-linguistic motivation per se, while this is less true for the encoding of, for instance, pragmatic nuances.

However, the claim of a principled autonomy in the language structure is only partially true in the case of EM, because of the role played by iconicity and iconic motivation in the structuring of the acoustic signal, which has been emphasised in the literature (see Hinton, Nichols and Ohala 1994a for a survey). In fact, it has been suggested that the phonosymbolic association of phonological features such as [high], [palatal] and so on with semantic features such as [small], [familiar] and related meanings, and of phonological features such as [low], [open] and so on with meanings such as [big], [hostile] etc., especially in contrast with morphemes displaying the former traits, may have a certain socio-cultural 
background. Ohala $(1994,1997)$ considers this association as due to the particular form taken by the mouth during the articulation of the respective sound types. In fact, the face assumes a smile-like expression with the mouth channel strengthened and thin during the articulation of palatal vowels, which usually display high values of $\mathrm{F}_{0}$, the so-called fundamental frequency. The smile-like expression may be taken to encourage a pacific, non-aggressive interaction between speaker and hearer. Conversely, low and open vowels normally provided with low $\mathrm{F}_{0}$ values are articulated by enlarging the mouth with an apparently aggressive intent. A similar correspondence of facial expressions, possibly accompanied by concrete sounds, and type of interaction is also found in apes and other animals, which leads Ohala to lay down the hypothesis that this correspondence has provided the evolutionary basis for the development of a cultural feature. If Ohala is right - but it must be added that the evidence in support of this explanation is not watertight; cf. Diffloth (1994) - we can observe a link between the way language is structured and the external world. ${ }^{1}$ At any rate, besides this single - and debated - case, the Saussurean postulate of the conventionality of language prevents any other possible interaction.

\subsubsection{The role of gender}

In order to understand the role played by factors of a sociolinguistic nature in the usage of $\mathrm{EM}$, let us start by discussing one finding which is generally mentioned as resulting from the impact of social factors on language (see Berruto 1995, 43-4 for a discussion). On average, women are considered to employ more EM (with an attenuative function) than do male speakers. This is valid in qualitative terms, as for instance in Portuguese, in which it is conceivable for a woman to use a diminutivised form of the word for thanking, namely obrigad-inha 'thanks-DIM.F', while this is not possible for a man: *obrigad-inho 'thanksDIM.M' (see Cardona 2006, 65-7, among others). In quantitative terms, too, it has been found that, for instance, in Tzeltal women make more use of the diminutive suffix/particle Pala as a signal of positive-politeness endearment in conversation (Brown and Levinson $1987,109,251)$. This fact has to be seen in connection with the general tendency manifested by female speakers towards a pronounced politeness in speech situations (Lakoff 1975; Holmes 1995). However, the interpretation of this finding is quite controversial. On the one hand, this can be taken as a classic example of the effect of a socially determined factor influencing a linguistic variety, namely that spoken by women. Since biological sex or gender is a variable normally taken into consideration alongside age, social class and so on, it seems obvious to consider this as a plausible sociolinguistic factor. On the other hand, one can wonder what kind of explanation is provided by the consideration of this factor. To be sure, it is not the case that this finding explains why the girl I am talking to employs more diminutives than I normally do. In this sense, this finding only expresses a quantitative correlation of a probabilistic nature observed in a certain corpus - feminine gender É more diminutives - which in its turn is in search of an explanation. Notice incidentally that sociolinguistic findings generally refer to a quantitative dimension, pinpointing correlations between social and linguistic features. This means that any predictive statement extracted from quantitative findings is of a probabilistic nature and cannot be interpreted deterministically.

Explanations of the correlation can appeal to a number of different factors and principles which illustrate what kind of influence sociolinguistic variables can have on language. For instance, one might think that the correlation depends on the way certain cultures socialise the important tasks of transmitting the affective values of the group and of keeping a favourable communicative mood or interaction style, assigning such a burden to women. 
On the other hand, women might behave in this way because they want or have to stress their social role, which is - again in certain cultures - to be seen as more related to the care of interpersonal relations rather than to the mere exchange of information. Finally, one might even venture the hypothesis that women are generally rather insecure and therefore prefer to limit the reach of their claims. As can be easily imagined, this is not only strongly culture-dependent (it is not difficult to imagine a different - matriarchal - society in which women are normally endowed with the opposite properties). What is more, the alleged explanations share the same directionality: the linguistic behaviour is seen as the reflex of a social fact, which is generally of a socio-historical nature and is formalised in such abstract, loose (and we may add: often simplistic) terms that its explanatory power is not easy to test or to falsify. In this light, its weakness is not only constitutive (because explanations in social sciences cannot be of a strictly deductive-nomological character; see Lass 1997, 328-9) but also external to language, because there is no real causal relationship between the usage of EM and the expression of a certain social value (and in fact, the attenuative function can be fulfilled by the employment of several linguistic means, such as honorifics, formulaic expressions of politeness and so on). This also means that it seems highly improbable to come up with sociolinguistic universals similar to those normally employed in language typology (Berruto 1995, 44). On the other hand, the explanations provided so far are of a functional nature, in the sense that they point to a teleological (or rather: teleonomical; Dressler and Merlini Barbaresi 1994, 16) motivation which explains women's attitude as based on the social division of the society. In this light, the pronounced politeness manifested by women in their increased usage of EM responds to a precise social function (on a functional understanding of the social relations, see Mesthrie 2006, 476). In addition, EM serves this social function in perfectly adequate terms because it is able to encode a number of semantic nuances which have less to do with referential effects on the listeners' mind but warrant their immediate, that is context-situated, response at the level of the speech act (I will come back to this point later). An important additional factor mentioned above is identity: language is an important mean for stressing personal identity, and as such it is commonly used by any social group for its self-statement. In this regard, it remains an open question whether women can be considered a social group on a par with others such as teenagers, old people and so on (see Edwards 2009, 132-44 for a recent survey). ${ }^{2}$

\subsubsection{Age as a sociolinguistic variable}

Besides gender, another important factor influencing the usage of EM is undoubtedly age. This is true of any kind of child-oriented communication which has been observed in the literature. For instance, in baby talk the role of EM is essential from the earliest stages of acquisition, in the sense that adults employ substantially more EM when talking to children (baby talk, motherese, nursery-school teacherese and so on) and conversely that children's average usage of EM is quite high, especially in the initial stages of language acquisition (Clark 1993, 168-9; Dressler and Korecky-Kröll in this volume). Although diminutive formation has been observed to be among the very first morphological operations acquired by children (Ferguson 1977, 225), this is true of any sort of EM, not only that relating to the so-called diminutivum puerile (see Staverman 1953 for the term). In fact, the child develops quite early the ability of constructing analogical formations which testify the salience of patterns of EM, exploiting for instance the contrast between diminutives and augmentatives. In this example from Italian, Matteo at the age of three extracts an augmentative form, barbone, on the basis of the false diminutive barboncino (De Marco 2002): ${ }^{3}$ 
(1) Matteo and his mother are walking in the street:

Matteo: mamma quello è un barbone

'Mummy that is a poddle'

Mother: perché dici che è un barbone?

'Why do you say it is a poddle?'

Matteo: perché è un barboncino grande

'because it is a big poddle.DIM'

EM and in particular diminutive formation has even been claimed to be the only kind of word formation available to children in the very early pre-morphological stage. ${ }^{4}$ In this light, EM plays an important role in the process of speech accommodation, which is normally understood as the process of attuning the speaker's communicative style to the interlocutor (Giles and Powesland 1975). Since the adult and the child converge in the high usage of EM, this becomes an important expressive dimension for the child, not only to develop harmonious relations with the present audience but also to explore the world outside of the usual participants. Let us give one brief example of the baby talk typically adopted by Viennese adults to address children. In this variety, a specific 'childish' suffix $-i$ (and its variants $-l i$, -tschi) is used, which has the property of leaving unaltered the property of the base (gender, word class), in contrast with other German diminutive suffixes such as -chen and -lein that form only neuter nouns (cf. respectively Bruder 'brother.M' $\rightarrow$ Brüder-chen 'little brother-DIM.NTR', Tisch 'table.M' $\rightarrow$ Tisch-lein 'small table-DIM.NTR', and so on). The childish suffix can be combined generally with nouns (Mama 'mum' $\rightarrow$ Mami, Papa 'daddy' $\rightarrow$ Papatschi) and with other word classes, as in these examples from motherese, in which the suffix is combined with adjectives and even verbs (Dressler and Merlini Barbaresi 1994, 106): ${ }^{5}$

(2) a. Guti guti. Bist doch g'scheitig'scheiti good:ie good:ie are.2sG yet clever:ie clever:ie 'Goody goody. You're a nice little clever child.'

b. Kindi muß brav sitzi, soll jetzt trinki child:ie must well-behaved sit:ie shall now drink:ie 'My little baby must now behave, sit quiet and drink his nice milk, come on, darling.'

Notice that the suffix is generally used by adults only with items that the child already knows, or, better, that the adults believe he or she knows (Dressler and Merlini Barbaresi 1994, 105). Moreover, the suffix cannot be followed by further inflectional endings; thus the verb remains uninflected and appears in the so-called inflective form (Teuber 1998), also typical of other language varieties such as those used in comics, chat-lines and so on.

As can be gathered from the translation, English, which has a very poor EM, displays a similar 'childish' suffix $-y /$-ie, enjoying a certain productivity normally restricted to child language or to situations metaphorically recreating a child's world, especially in hypocoristics (Johnn-y, Kat-ie and so on), kinship terms (mumm-y, grann-y and so on) and other nouns such as dogg-ie, bunn-y, and even adjectives such as fatt-y, short-ie. This suffix has also been reported to be typically used by women in expressions such as: Give (me) your hand-y! (Charleston 1960, 123). Since older people are frequently treated like infants by their caregivers (Ferguson 1977, 230), Dressler and Merlini Barbaresi (1994, 190-1) 
introduce the term diminutivum senile for the following Italian example, in which an adult woman reproaches an elderly man complaining about the small amount of food he has received: 6

$$
\begin{aligned}
& \text { Avete anche mangiato un ovino } \\
& \text { have.2PL also eaten an egg.DIM } \\
& \text { 'You've even had egg to eat!'. }
\end{aligned}
$$

However, they observe that there seem to be culture-specific socio-pragmatic restrictions involved here, because the use of the diminutivum senile is allegedly blocked in similar situations in Arabic and Chinese because of great respect for old age. Nevertheless, even in these languages this restriction is effaced when speaking about very old, dependent persons in their absence.

The role played by diminutives is also important because it illustrates how the primary socialisation of children takes place, by emphasising a smooth approach to our daily experience in which attenuative tuning creates the conditions for a harmonious relation with the adults' world as a normal expectation. In this regard, it has been observed that this smooth interaction with children characterises humans and to a lesser extent apes as distinct from other species (Tomasello 1999). This attitude largely emerges within mother-child relations and has to be looked upon in connection with women's privileged usage of diminutives discussed above, taking especially into consideration their role as a primary agent in the children's developmental path. Notice that the crucial role played by EM in child-centred speech situations is also important for the understanding of its usage in speech situations characterised by the non-serious attitude of the speakers involved (see Section 2.2).

In this light, it does not come as a surprise to observe that high usage of EM also characterises young people as a social group. In fact, 'the peer group (e.g., the child's circle of playmates within and outside the home) soon overtakes the parents as the main reference group in transmitting linguistic behavior' (Mesthrie 2006, 476). In this connection, it has been emphasised that the high usage of evaluative expressions, besides their expressive strength, has the specific intent of signalling identity as a social group. This is, for instance, clearly the case with new varieties resulting from the multiethnic context characterising the urban development of several European capitals, in which new varieties of youth language have developed in recent years, such as Kiezdeutsch in Berlin (Wiese 2012), Rinkeby Svenska in Stockholm (Kotsinas 1998) or Multicultural London English (Kerswill 2006). These varieties present similar phenomena in particular with regard to the large use of $\mathrm{EM}$, sometimes displaying specific features distinct from other language varieties because of the contact context. For instance, Multicultural London English uses the word nang to express high appreciation: Last night was so nang! The source of the term is unclear, perhaps of foreign (Bengali, Persian) origin. This gives rise to further formations, such as the reduplication nangnang referring to a 'person who is prone to doing foolish things' or to an 'edible pick-me-up usually desired after a long night of drinking and smoking up'; the derivatives nangage 'moment of satisfaction', nangidge 'something cool', nangified 'especially nang'; the blends nangtastic 'really cool', nangry 'super angry', nanguin based on penguin and generally used as a pet name; and so on. ${ }^{7}$ Notice the pronounced usage of extra-grammatical techniques which aim at the effect of hyperbolisation typical of the language of youth (Augenstein 1998, 147). This can also be attained by using evaluative expressions as modifiers in compounds: nang skunk 'really good weed'; especially 
in languages where the latter are very productive, as in German: arschgeil 'really cool, lit. ass cool', saugenial 'really brilliant, lit. sow genial', and even with multiple modification: superarschviel 'very much, lit. super ass much', oberaffentittengeil 'really cool, lit. over monkey hooters cool' and so on (Androutsopoulos 1998, 117). Notice that the modifiers arsch-, sau- display an utterly positive evaluation in contrast with their normal value, outside of the youth language, which has a negative connotation: arschkalt 'very cold', sauteuer 'very expensive' and so on (Androutsopoulos 1998, 110). Similar to what happens in child language, special 'youngish' suffixes are also used, for instance in the German shortenings Alkoholiker 'heavy drinker, alcoholic' $\rightarrow$ Alki, Zigarette 'cigarette' $\rightarrow$ Ziggi, Prolet(arier) 'redneck' $\rightarrow$ Prollo, depressiv 'depressive' $\rightarrow$ depro, and so on (see Androutsopoulos 1998, 118-24).

Finally, a particular interesting case of interaction between the factors of age and gender can be drawn from Italian, in which the rich inventory of EM is especially exploited in computer-mediated communicative contexts such as blogs, chat-lines and so on:

$$
\begin{aligned}
& \text { E poi Benafflec. Lui, che normalmente ha quest'aria da sallocchione, belloccio } \\
& \text { ma proprio sedano. Lui che beve un uischi torbato ma pensa a GenniferGarniè } \\
& \text { (la donna senza sopracciglia). Insomma, di solito è bello ma non è che mi } \\
& \text { entusiasmi. Invece in Argo è un sorcone strappamutande che manco ci posso } \\
& \text { ripensare. (http://comesefossebionda.wordpress.com/tag/cimena) }
\end{aligned}
$$

'And then Ben Affleck. He normally looks like a real klutz (lit. a sausage.AUG), pretty.DIM but really silly (lit. celery). Drinking a peaty whisky while thinking about Jennifer Garner (the woman without eyebrows). In short, he's usually pretty but I'm not really fond of him. In contrast, in Argo he's really so cute (lit. a rat. AUG.M who strips off underwear) that I can't stop thinking about him.'

In particular, the last vivid expression nicely shows how far the expressive power of EM can go, because the base of sorcone, the feminine noun sorca 'rat' then 'feminine sexual organ', is normally used by (Roman) male speakers to indicate a showy, provoking and sexually desirable woman. In contrast, '[g]irls use the neologism sorcone for "charming and good looking boy", based in its turn on the masculine conversion sorco (Romiti 1998, 291). In other words, the usage of such a term immediately qualifies the speaker or writer as provided with the features [young] and [female]. ${ }^{8}$

\subsubsection{Evaluative morphology and diaphasic variation}

Moving to the diaphasic dimension, it has been claimed several times that spoken or informal varieties display a higher frequency of EM than written or controlled varieties (in this regard, recall Grandi's 2003a findings concerning the Italian augmentative suffix -one, mentioned above). Moreover, this is clearly true of all those situations in which affective relations are involved (for example, pet- and lover-centred speech situations), because the latter also often reproduce child-centred speech situations (Dressler and Merlini Barbaresi 1994, 191-7). As for more controlled registers, it has been often emphasised that EM generally occurs seldom in written varieties of specialized language (Roelcke 1999, 74). However, this does not exclude the possibility that single instances of EM can be used even in controlled registers, generally for terminological reasons. Moreover, specialized language also displays vertical variation, going from more to less controlled registers. This makes it difficult to postulate categorical rules such as that suggested by Rainer (1989, 212) for Italian, which alleges that no diminutives can be formed from nouns belonging to 
bureaucratese or to abstract, scientific or other high-brow lexical domains (5a), while the restriction weakens with bases used in more colloquial contexts $(5 b)$ :

(5) a. alunno 'disciple' $\rightarrow$ *alunn-ino/*alunn-etto 'disciple-DIM' allievo 'student' $\rightarrow$ *alliev-ino/*alliev-etto 'disciple-DIM'

b. scolaro 'disciple' $\rightarrow$ scolar-etto 'disciple-DIM' studente 'student' $\rightarrow$ student-ello 'student-DIM'

As a matter of fact, the vertical variation observed in the different Italian registers has the effect of providing a whole range of expressions displaying diminutivised forms in private texts, not necessarily only in ludic contexts (6a) but also in more controlled varieties of specialized language such as the official documents of an elementary school (6b) (examples from the Internet):

(6) a. l'anno prossimo la mia classe andrà in terza (elementare) [. . . vorrei intraprendere con i miei alunnetti un laboratorio sulle emozioni e l'autostima (http://www.maestrasabry.it/forum/viewtopic.php? $\mathrm{f}=19 \& \mathrm{t}=125$ ) 'next year my class will attend the third year (of the elementary school) [. . . ] I would like to start with my disciples.DIM a laboratory on emotions and self-confidence'

b. La scuola è raccordata con Enti, Istituzioni e genitori degli alunnini per la realizzazione delle attività (http://csakr.calabriascuola.it/allegati\%5Cnews\%5C121\%5CDON\%20 MATTEO\%20LAMANNA\%20-\%20MESORACA.do)

'The school is linked with authorities, institutions and parents of the disciples. DIM for the realization of the activities.'

The same can be observed for the Italian elative suffix -issimo (Rainer 2003), which is predicted not to be combined with adjectives belonging to high-brow lexical domains, for instance significativo 'significant'; but Rainer himself finds one example of significativissimo in a literary essay, and some more on the Internet.

In sum, it is probably more adequate to speak of Labovian variable rules depending on several factors such as frequency rank of the derivatives and of their bases, text genre, situational context and so on, instead of categorical restrictions. Finally, socio-cultural factors can also have an effect: for instance, Dressler and Merlini Barbaresi $(1994,215)$ report the following case of diminutivum modestum drawn from the Italian academic discourse uttered by a (female) linguist in the discussion period of a large conference: Avrei anch 'io una mia teorietta in proposito 'I'd have a theory.DIM myself on this point.' These authors comment that such a situation would be much too formal to allow a diminutive in German. In other words, the reduction of social and psychological distance effected by the use of EM is strictly related to the general mechanisms underlying the so-called interactional discourse active in a given language, which 'has as its primary goal the establishment and maintenance of social relationship' (Kasper 1990, 205). In this regard, Dressler and Merlini Barbaresi $(1994,216)$ go on to observe that, according to their informants, Italian sides with Slovene and Spanish while Czech and Basque behave rather like German. Similarly, Italian political and economic discourse tolerates a far larger use of EM in contexts in which no ludic effect is attained, as testified by examples such as an economic ripres-ina 
'recovery-DIM', the government's firm-etta 'signature-DIM' for one particularly demanding decret-one 'decree-AUG', the concors-accio 'contest-PEJ' to hire new school teachers, and so on. Finally, the importance of modulating the degree of social or psychological distance in Italian is shown by the following dialogue between a female customer (C) and the male owner (O) of a shoe shop (Dressler and Merlini Barbaresi 1994, 217):

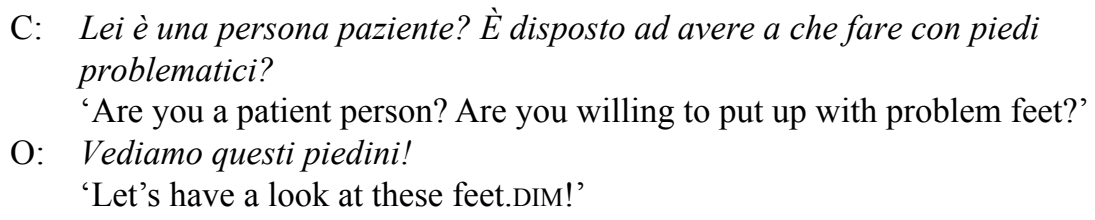
problematici?

'Are you a patient person? Are you willing to put up with problem feet?'

O: Vediamo questi piedini!

'Let's have a look at these feet.DIM!'

In spite of the clearly asymmetrical customer-seller relation, requiring a certain degree of formality and social distance, the owner reacts to the non-conventional manner adopted by the customer by using a diminutive form in order to reduce the distance while giving a ludic character to the speech situation.

\subsection{Evaluative morphology in the socio-pragmatic dimension}

Let us briefly turn our attention to the opposite perspective, namely what kind of ingredients EM can offer in order to be exploited felicitously in the socio-pragmatic dimension. Here pragmatics comes necessarily into play because the perspective of language use is central for understanding the particular role of EM within a given speech situation (cf. Merlini Barbaresi in this volume). In this regard, it has already been emphasised that language contributes to personal and social identity. Terestyéni (1995) identifies three potential human character types: contact-oriented, status-oriented and task-oriented people, depending on their preference for 'the connection-creating' or 'the power-representative style of knowledge'. Among others, one type of feature distinguishing the three character types relates to the way people establish personal relationships: whether they choose friends according to their common personal experiences, or to their social status, or finally to their common (for example, professional) interests. It seems plausible to assume that contact-oriented people are likely to make a larger use of EM than the other two. ${ }^{9}$ This is due to the relevant feature [non-serious], which can be assigned to EM in a morphopragmatic understanding of its value as 'a natural meaning extension of [small]' (Dressler and Merlini Barbaresi 1994, 398; Jurafsky 1996). In this connection, Spitzer's (1921, 201-2) pioneering remarks can be mentioned: 'Der Spieltrieb ist eine Stimmung im Sprecher, die die Grundierung des Satzes, die "key" abgibt [. . . ] Die Suffixe wirken wie Vorzeichen in der Musik, sie bestimmen die "Tonart" der menschlichen Rede.' 10

The ludic perspective opened up by the use of EM makes it highly suitable for the pragmatic operations of downgrading and mitigation, facilitating social contact (Caffi 2007, 99-100). In this sense, EM markers function as personal and social indices to refer to relevant elements in the speech situation anchoring the speakers to their specific roles and responsibilities in the society (Levinson 1983, 89-94). To take one example, if it may appear obvious that EM contributes to downgrading and mitigation; it is less evident that the use of diminutives may also imply upgrading, inasmuch as the speakers involved in the speech act are compelled to respond politely to mitigated requests exactly because they are formulated in a downgraded way. Therefore, they would lose face by a refusal. An Italian speaker is well aware that a request containing EM such as Mi stamperesti queste 
due paginette? 'Would you print these two pages.DIM?' is essentially more binding than its unmarked variant, because a refusal requires a stronger stance by the listener (Dressler and Merlini Barbaresi 1994, 248). On the other hand, the diminutive form can be used to reproach the addressee insofar as she or he is equated to a child, which in a predominantly masculine society with its traditional (over-)emphasis on the notions of manliness and machismo can be felt as a face-threatening insult. In the following example from Colloquial Jordanian Arabic (Badarneh 2010), a mother addresses her eighteen-year-old son, complaining of his immaturity and irresponsibility:

$$
\begin{aligned}
& \text { yaa xwaylid quum udrus } \\
& \text { vOC xaalid.DIM stand:up study.IMP } \\
& \text { 'Khalid.DIM, go and study [for your exams]!' }
\end{aligned}
$$

The effect is crucially connected with the use of an allocutive form - in this case the proper name - containing EM, as will be discussed below. This example also shows that the employment of EM as a morphopragmatic marker is subject to restrictions of a sociocultural and ethnographic nature, as for instance this use contrasts with the marking of the diminutive on an adult's proper name (for example, John vs. Johnnie) in English and other languages to express affection and endearment, which is oriented towards one's positive face (Travis 2004). In this regard, it has already been observed above that certain cultures tolerate the use of EM in certain speech situations while others do not, which also implies a learning process for new members (children and foreigners; cf. Dressler and Korecky-Kröll in this volume). This may also lead to socio-cultural clichés which are on one hand arbitrary and stereotypical, while on the other they serve as a powerful means of self-identification and affirmation as a community. For instance, the abundance of EM displayed by Slavic, Baltic and Mediterranean people has been opposed as the manifestation of an 'excessive' and somewhat 'childish' attitude to British 'soberness' or to clarté française (Dressler and Merlini Barbaresi 1994, 409-10). ${ }^{11}$

In this connection, it does not come as a surprise to observe that EM plays a role in languages with fully fledged systems of honorifics, the latter intended as 'resources for indexing the relative position of interlocutors, referents and bystanders either in the lexicon or the morpho-syntax of a language' (Brown 2011, 19). As is well known, honorifics are a central part of social deixis and pay particular attention to those factors of a sociolinguistic nature (for instance, age, wealth, occupation, family background) that determine categories of people and their interactions, and generally comprise a complex system of expressive tools going far beyond word formation (see Shibatani 2006 for a survey). For our purposes, besides the sparse reference to (largely opaque) etymologies of single honorific affixes going back to evaluative expressions, such as the all-purpose Japanese honorific prefix $o$ - used with native nouns like o-kaban 'bag', o-hanasi 'talk' and so on, which is associated with the meaning 'greatness' (Shibatani 2006, 382), a clear-cut case of EM with an honorific value is provided by the Nahuatl diminutive suffix -tzin (Hill and Hill 1978). Depending on the context, this suffix can be used as a truly diminutive suffix, or to mark reverential address as a distance-respect marker, or as a form for expressing endearment and affection. Thus, while it normally marks the relationship holding among the speech participants and can be used with any kind of lexeme including particles and adverbs (for instance, ämo-tzīn 'no-DIM' and ihcōn-tzìn 'thus-DIM'), especially in highly reverential speech situations involving older people ('compadres', the godfathers), parent terms such as 'mother' and 'father' cannot be used without the diminutive suffix in everyday speech 
(see for instance the reverential expression ina-huac-tzin in no-tah-tzin 'with my revered father, lit. his-with-DIM the my-father-DIM', in which the preposition also carries the diminutive suffix), unless they occur in insults: pütoh mitztläcatilīh in mo-nānah 'son of a bitch!, lit. whore she:bore:you the your-mother'. Notice incidentally that this easily leads to lexicalised expressions such as tlācatzīntli 'gentleman' from tlācatl 'man', zoātzīntli 'lady' from zoātl 'woman', àtzintli 'holy water' from àtl 'water', and so on. On the other hand, a form such as no-nān-tzīn 'my-mother-DIM' can be interpreted as a highly respectful address form: 'my reverend mother', or, equally well, as a more intimate 'my dear little mother', or as both, depending on the context.

The [non-serious] feature attributed to a speech act with the help of EM is largely supported by the particular flexibility of EM, which lends itself to several uses normally impossible with other word-formation patterns. One example of this flexibility is the low categoriality which is testified by the selection as a landing site of unexpected parts of speech, as in the Nahuatl examples discussed above involving particles, adverbs and prepositions, or in the case of the Spanish gerund: calland-ito 'being quiet-DIM', corriendito 'running-DIM' and so on (NGLEM 2010, 512):

Debíamos de acercarnos callandito - sugería Fernando.

'We have to get close keeping quiet - Fernando suggested.'

One further peculiar property of EM is the occurrence of so-called interfixes, namely linking elements accompanying an evaluative morpheme and possibly adding semantic nuances which are relevant at the morphopragmatic level (cf. Merlini Barbaresi, this volume). For instance, Rainer (1993, 161-70) describes the complex system of peculiar allomorphies displayed by evaluative suffixes in Spanish, which to a certain extent also involves truly sociolinguistic variables such as urban/non-urban origin (as in the proper name Juan-ito vs. Juan-cito), common/technical term (as in the feminine nouns mano 'hand' $\rightarrow$ man-ita vs. moto 'motorbike' $\rightarrow$ mot-ito), and so on.

Finally, a particularly striking case in which EM is used to encode sociolinguistic information, exploiting its flexibility, is found in Nootka (and other North American languages such as Yana; Sapir 1915). Here, the diminutive suffix - 'is attached to the verb form is normally used for addressing children (10a) or for talking about children (10b) or even for speaking about oneself when addressing a child, but only to show affection at the same time (10c):

(10) a. qwis-'is-tcic

do:so-DIM-2SG.IMP

'do so, little one!'

b. qwis-'is-mac

do:so-DIM-3SG.PRS.IND

'He does so'

c. wat-ciL-'is-aH

go:home-INCP-DIM-I

'I'm going home, little one!'

Interestingly, the same diminutive suffix identifies social groups such as small people or dwarfs (11a), in this case accompanied by a palatalisation of all sibilants: $s \rightarrow \dot{s}, t s \rightarrow t^{\prime}$ 
and so on; eye-impaired (not blind!) people (11b), with the addition of the lateralisation of sibilants: $s \rightarrow \ell, t s \rightarrow L$ and so on; hunchbacks (11c), this time with the sibilants becoming 'thickish' sounds pronounced with the lower jaw held in front of the upper; left-handed people (11d), with the addition of the meaningless element $-t c H^{a}$ - inserted after the first syllable; and so on:

(11)
a. hin-tc-sil-'iś-we'in
LIGHTV-COMe-INCP-DIM-QUOT
'he, little man, comes, they say'
b. qwit $^{\prime}$ 'it-mac
do:so-DIM-3SG.PRS.IND
'he, one-eyed man, does so'
c. yátcc-uk $k^{c}-i c-m a^{c}$
walk-INTR-DIM-3SG.PRS.IND
'he, hunchback, is walking'

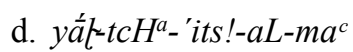
be:there-EP-DIM-now-3SG.PRS.IND
'there now he is, poor little left-handed chap!'

This variety of uses stresses the value of EM as a marker for expressing social relations and socio-pragmatic connotations within a speech community. In this regard, Sapir [1915] (1991, 362) observes that 'these distinctive forms are generally avoided when in the presence of [the referred] people, for fear of giving offence'. As explained throughout this chapter, the speaker must have access to a large number of pieces of information of a linguistic and social nature in order to become acquainted with the behavioural norms valid within a speech community. Nootka shows masterfully how their richness is perfectly covered by the large expressive potential of EM.

\section{Notes}

1. Dressler (1994) sees the reason underlying the iconic structuring of EM in its early development in small children, at a stage when no distinction is made between grammatical rules and the socalled extra-grammatical (or 'expressive') morphology which does not obey a strict rule-based pattern. Ohala's hypothesis provides the phylogenetic background for Dressler's ontogenetic interpretation. See the discussion below.

2. In this regard, cf. Cameron and Coates' conclusion (1988, 24): 'Explaining sex differences does not just mean explaining the usage of women, after all. It means devising methods applicable to all informants, so we can gauge the importance of sex in the complex system of intersecting social relations that support linguistic variation.'

3. Notice that barboncino is a false diminutive based in its turn on the false augmentative barbone, which actually means 'beggar' from barba 'beard', lit. 'long beard'. Clearly, Matteo's creation is not influenced by the derivational history of the base, which is apparently unknown to him.

4. Cf. Dressler and Merlini Barbaresi (1994, 408): 'Diminutive formation is the first morphological rule that small children acquire [. . .], that is, it is acquired at a time when there is no distinction yet between extragrammatical morphological operations and grammatical rules.' 
5. This 'childish' suffix differs from the its homonym $-i$ occurring in formations such as Studi 'student', Fundi 'fundamentalist' and so on, because it is not restricted to baby talk and generally forms nouns (Androutsopoulos 1998, 118-21).

6. Notice the use of the verbal form in the second person plural, which is normal in several varieties of Italian for addressing persons deserving a certain respect, such as older people.

7. For information see http://www.urbandictionary.com.

8. At any rate, since the Italian augmentative suffix -one can also give rise to masculine nouns from feminine bases, such as donna 'woman.F' $\rightarrow$ donn-one 'woman-AUG.M', sorcone can also be used, although in a less conventionalised manner, by male speakers with reference to attractive women, as in this example from the Internet: Incontrata 20 giorni fa agli imbarchi di Fiumicino. Un sorcone monumentale. . 'Met.F 20 days ago in the boarding area of Fiumicino airport. A monumental rat.AUG.M'.

9. In this regard, recall what has been said above about women, who on average use more diminutives and are taken to exert a social function oriented towards the maintenance of social relationships, for instance solidarity (Holmes 1995, 11-16).

10. "The ludic instinct is a mood of the speaker's which creates the ground of the sentence, the "key" [...] Suffixes [sc. diminutives] work like key signatures in music, determining the "key" of human speech.'

11. National clichés lead to the hyper-characterisation of certain features, often with ironic intent, by bilingual foreigners, as shown for instance by the exaggerated and inappropriate use of diminutives made by Voltaire when writing Italian (for example, bigliettini 'tickets.DIM' for theatre tickets, while the term normally refers to a note) while he was very sparing of this in French (Folena 1983, 410). In France, as a reaction against Italian influence, the use of EM has been largely condemned since the seventeenth century, leading to a decay of its use in the standard variety (Hasselrot 1957, 216). 